\title{
Exponential Stability for Switched Systems with Mixed Time Delays
}

\author{
Chang-Hua Lien ${ }^{*}$,, Ker-Wei Yu ${ }^{\mathrm{a}}$, Yen-Feng Lin ${ }^{\mathrm{a}}$, Yeong-Jay Chung ${ }^{\mathrm{a}}$, Long-Yeu Chung ${ }^{\mathrm{b}}$ and \\ Jenq-Der Chen ${ }^{\mathrm{c}}$
}

\author{
${ }^{a}$ Department of Marine Engineering, National Kaohsiung Marine University, Kaohsiung, Taiwan 811, Republic of China \\ ${ }^{b}$ Department of Electrical Engineering, Tung-Fang Institute of Technology, Kaohsiung, Taiwan 829, Republic of China \\ ${ }^{c}$ Department of Electronic Engineering, National Kinmen Institute of Technology, Kinmen, Taiwan 892, Republic of China
}

\begin{abstract}
The global exponential stability problem for a class of switched systems with mixed time delays is investigated in this paper. LMI-based delay-dependent and delay-independent criteria are proposed to guarantee the exponential stability for our considered systems. Razumikhin-like approach and Leibniz-Newton formula are used to find the stability results. Finally, a numerical example is illustrated to show the improved results from using this method.
\end{abstract}

Keywords: Global exponential stability, switched neutral system, LMI-based approach.

\section{INTRODUCTION}

The time-delay phenomenon is encountered in many practical systems, such as aircraft stabilization, neural networks, nuclear reactors, ship stabilization, and systems with lossless transmission lines. It is well known that the existence of time delay in a system may cause instability or bad system performance in open and closed-loop systems. Hence stability analysis for time-delay systems has received considerable attention in recent years [1-3]. In many physical systems, the system models can be described by functional differential equation of neutral type, in which the models depend on the state of delay but also depend on state derivatives. Neutral system examples include distributed networks, heat exchanges, and processes including steam. Stability conditions have been proposed to guarantee the stability for neutral systems [4-5]. On the other hand, stability for switched time-delay systems has been an attractive research topic which is composed of subsystems with their own parameterizations [6-7]. Hence we will consider the stability problem for switched neutral systems in this paper. Based on Razumikhin-like approach and Leibniz-Newton formula [8], delay- dependent and delay-independent results are provided. The LMI approach [9] is an efficient and powerful tool in solving some control problems; such as $H_{\infty}$ control, state feedback control, and observer-based control. Hence, we will recommend the LMI-based results. A numerical example is provided to demonstrate the proposed results.

The notation used throughout this paper is as follows. For a matrix $A$, we denote the transpose by $A^{T}$, spectral norm by $\|A\|$, symmetric positive (negative) definite by $A>0$ $(A<0) . A \leq B$ means that matrix $B-A$ is symmetric

*Address correspondence to this author at the Department of Marine Engineering, National Kaohsiung Marine University, Kaohsiung, Taiwan 811, Republic of China; Tel: 886-7-8100888, Ext. 5223; Fax: 886-7-5718302; Email: chlien@mail.nkmu.edu.tw positive semi-definite. For a vector $x$, we denote the Euclidean norm by $\|x\|$ and $\left\|x_{t}\right\|_{s}=\sup _{-H \leq \theta \leq 0} \sqrt{\|x(t+\theta)\|^{2}+\|\dot{x}(t+\theta)\|^{2}}$. $I$ denotes the identity matrix.

\section{PROBLEM FORMULAION AND MAIN RESULTS}

Consider the following switched neutral system with mixed time delays:

$$
\begin{aligned}
& \dot{x}(t)-D \dot{x}(t-\tau)=A_{0 \sigma} x(t)+A_{1 \sigma} x(t-h(t)), t \geq 0, \\
& x(t)=\phi(t), \quad t \in[-H, 0],
\end{aligned}
$$

where $x \in \mathfrak{R}^{n}, \quad x_{t}$ is state at time $t$ defined by $x_{t}(\theta):=x(t+\theta), \quad \forall \theta \in[-H, 0], \quad \sigma \quad$ is a switching signal which is a piecewise constant function and may depend on $t$ or $x, \sigma$ takes its values in the finite set $\{1,2, \cdots, N\}$, timevarying delay $0 \leq h(t) \leq h_{M}, \dot{h}(t) \leq h_{D}, h_{M}>0, \tau>0$, $H=\max \left\{h_{M}, \tau\right\}$. Matrices $D, A_{0 i}$, and $A_{1 i} \in \mathfrak{R}^{n \times n}$, $i=1,2, \cdots, N$, are constant, and the initial vector $\phi \in C_{1}$, where $C_{1}$ is the set of differentiable functions from $[-H, 0]$ to $\Re^{n}$.

Define the functions $\lambda_{i}(t), i=1,2, \cdots, N$, in the following:

$\lambda_{i}(t)=\left\{\begin{array}{cc}1, & \sigma=i, \\ 0, & \text { otherwise, }\end{array} \quad i=1,2, \cdots, N\right.$.

The switched system in (1) can be rewritten as follows:

$\dot{x}(t)-D \dot{x}(t-\tau)=\sum_{i=1}^{N} \lambda_{i}(t)\left[A_{0 i} x(t)+A_{1 i} x(t-h(t))\right], t \geq 0$,

$x(t)=\phi(t), \quad t \in[-H, 0]$,

where $\lambda_{i}(t)$ is defined in (2) and $\sum_{i=1}^{N} \lambda_{i}(t)=1, \forall t \geq 0$. 
Definition 1: The system (1) is said to be the globally exponentially stable with convergence rate $\alpha>0$, if there are two positive constants $\alpha$ and $\Psi$ such that

$\|x(t)\| \leq \Psi \cdot e^{-\alpha t}, \quad t \geq 0$.

Now we present a delay-dependent condition for the global exponential stability of system (1) with (2).

Theorem 1: System (1) is global exponentially stable with convergence rate $0<\alpha<-(\ln \|D\|) / \tau$, if $\|D\|<1$ and there exist some $n \times n$ matrices $P, Q, R, S, T>0$, a matrix $U$, such that the following LMI conditions hold for all $i=1,2, \cdots, N$

$\Sigma_{i}=\left[\begin{array}{ccccc}\Sigma_{11 i} & \Sigma_{12 i} & \Sigma_{13 i} & \Sigma_{14 i} & \Sigma_{15 i} \\ * & \Sigma_{22 i} & \Sigma_{23 i} & 0 & 0 \\ * & * & \Sigma_{33 i} & \Sigma_{34 i} & \Sigma_{35 i} \\ * & * & * & \Sigma_{44 i} & \Sigma_{45 i} \\ * & * & * & * & \Sigma_{55 i}\end{array}\right]<0$,

where * represents the symmetric form in the matrix and

$\Sigma_{11 i}=2 \alpha \cdot P+P A_{0 i}+A_{0 i}^{T} P+Q+S-e^{-2 \alpha h_{M}} \cdot R$,

$\Sigma_{12 i}=-2 \alpha \cdot P D-A_{0 i}^{T} P D, \Sigma_{13 i}=P A_{1 i}+e^{-2 \alpha h_{M}} \cdot R$,

$\Sigma_{14 i}=A_{0 i}^{T} U^{T}, \Sigma_{15 i}=-A_{0 i}^{T} U^{T} D$,

$\Sigma_{22 i}=2 \alpha \cdot D^{T} P D-e^{-2 \alpha \tau} \cdot S, \Sigma_{23 i}=-D^{T} P A_{1 i}$,

$\Sigma_{33 i}=-e^{-2 \alpha h_{M}} \cdot\left[\left(1-h_{D}\right) \cdot Q+R\right], \Sigma_{34 i}=A_{1 i}^{T} U^{T}$,

$\Sigma_{35 i}=-A_{1 i}^{T} U^{T} D, \Sigma_{44 i}=T+h_{M}^{2} \cdot R-U-U^{T}$,

$\Sigma_{45 i}=\left(U+U^{T}\right) D$,

$\Sigma_{55 i}=-D^{T}\left(U+U^{T}\right) D-e^{-2 \alpha \tau} \cdot T$.

Proof: Define the Lyapunov functional

$V\left(x_{t}\right)=e^{2 \alpha t} \cdot(x(t)-D x(t-\tau))^{T} P(x(t)-D x(t-\tau))$

$+\int_{t-h(t)}^{t} e^{2 \alpha s} \cdot x^{T}(s) Q x(s) d s$

$+h_{M} \cdot \int_{t-h_{M}}^{t} e^{2 \alpha s} \cdot\left(s-\left(t-h_{M}\right)\right) \dot{x}^{T}(s) R \dot{x}(s) d s$

$+\int_{t-\tau}^{t} e^{2 \alpha s} \cdot x^{T}(s) S x(s) d s+\int_{t-\tau}^{t} e^{2 \alpha s} \cdot \dot{x}^{T}(s) T \dot{x}(s) d s$,

where $P, Q, R, S, T>0$. The time derivatives of $V\left(x_{t}\right)$, along the trajectories of system (3) satisfy

$$
\begin{aligned}
& \dot{V}\left(x_{t}\right)=e^{2 \alpha t} \cdot\left[2 \alpha \cdot(x(t)-D x(t-\tau))^{T} P(x(t)-D x(t-\tau))\right] \\
& +e^{2 \alpha t} \cdot \sum_{i=1}^{N} \lambda_{i}(t)\left[\left(A_{0 i} x(t)+A_{1 i} x(t-h(t))\right)^{T} P(x(t)-D x(t-\tau))\right] \\
& +e^{2 \alpha t} \cdot \sum_{i=1}^{N} \lambda_{i}(t)\left[(x(t)-D x(t-\tau))^{T} P\left(A_{0 i} x(t)+A_{1 i} x(t-h(t))\right)\right]
\end{aligned}
$$

$$
\begin{aligned}
& +e^{2 \alpha t} \cdot\left[x^{T}(t) Q x(t)-(1-\dot{h}(t)) \cdot e^{-2 \alpha h(t)} \cdot x^{T}(t-h(t)) Q x(t-h(t))\right] \\
& +e^{2 \alpha t} \cdot\left[h_{M}^{2} \cdot \dot{x}^{T}(t) R \dot{x}(t)-h_{M} \cdot \int_{t-h_{M}}^{t} e^{2 \alpha(s-t)} \cdot \dot{x}^{T}(s) R \dot{x}(s) d s\right] \\
& +e^{2 \alpha t} \cdot\left[x^{T}(t) S x(t)-e^{-2 \alpha \tau} \cdot x^{T}(t-\tau) S x(t-\tau)\right] \\
& +e^{2 \alpha t} \cdot\left[\dot{x}^{T}(t) T \dot{x}(t)-e^{-2 \alpha \tau} \cdot \dot{x}^{T}(t-\tau) T \dot{x}(t-\tau)\right] .
\end{aligned}
$$
have

By the inequality of [1] and Leibniz-Newton formula, we

$$
\begin{aligned}
& -h_{M} \cdot \int_{t-h_{M}}^{t} e^{2 \alpha s} \cdot \dot{x}^{T}(s) R \dot{x}(s) d s \\
& \leq-h(t) \cdot e^{2 \alpha t} \cdot e^{-2 \alpha h_{M}} \cdot \int_{t-h(t)}^{t} \dot{x}^{T}(s) R \dot{x}(s) d s \\
& \leq-e^{2 \alpha t} \cdot e^{-2 \alpha h_{M}} \cdot\left[\int_{t-h(t)}^{t} \dot{x}(s) d s\right]^{T} R\left[\int_{t-h(t)}^{t} \dot{x}(s) d s\right] \\
& =-e^{2 \alpha t} \cdot e^{-2 \alpha h_{M}} \cdot[x(t)-x(t-h(t))]^{T} R[x(t)-x(t-h(t))] .
\end{aligned}
$$

By system (3), we have

$$
\begin{aligned}
& -(\dot{x}(t)-D \dot{x}(t-\tau))^{T}\left(U+U^{T}\right)(\dot{x}(t)-D \dot{x}(t-\tau)) \\
& +\sum_{i=1}^{N} \lambda_{i}(t)\left[(\dot{x}(t)-D \dot{x}(t-\tau))^{T} U\left(A_{0 i} x(t)+A_{1 i} x(t-h(t))\right)\right] \\
& +\sum_{i=1}^{N} \lambda_{i}(t)\left[\left(A_{0 i} x(t)+A_{1 i} x(t-h(t))\right)^{T} U^{T}(\dot{x}(t)-D \dot{x}(t-\tau))\right]=0 .
\end{aligned}
$$

By (6a)-(6c), we obtain the following result

$$
\dot{V}\left(x_{t}\right) \leq e^{2 \alpha t} \cdot \sum_{i=1}^{N} \lambda_{i}(t) \cdot\left[X^{T} \cdot \Sigma_{i} \cdot X\right]
$$

where

$X^{T}=\left[\begin{array}{lllll}x^{T}(t) & x^{T}(t-\tau) & x^{T}(t-h(t)) & \dot{x}^{T}(t) & \dot{x}^{T}(t-\tau(t))\end{array}\right]$, matrices $\Sigma_{i}, i=1,2, \cdots, N$, are defined in (4). From the conditions $\Sigma_{i}<0, i=1,2, \cdots, N$, in (4), we have

$V\left(x_{t}\right) \leq V\left(x_{0}\right), t \geq 0$,

where

$$
\begin{aligned}
& V\left(x_{0}\right)=(x(0)-D x(-\tau))^{T} P(x(0)-D x(-\tau)) \\
& +\int_{-h(0)}^{0} e^{2 \alpha s} \cdot x^{T}(s) Q x(s) d s \\
& +h_{M} \cdot \int_{-h_{M}}^{0} e^{2 \alpha s} \cdot\left(s+h_{M}\right) \dot{x}^{T}(s) R \dot{x}(s) d s \\
& +\int_{-\tau}^{0} e^{2 \alpha s} \cdot x^{T}(s) S x(s) d s+\int_{-\tau}^{0} e^{2 \alpha s} \cdot \dot{x}^{T}(s) T \dot{x}(s) d s \\
& \leq \delta_{1} \cdot\left\|x_{0}\right\|_{s}^{2}, \\
& \text { and } \\
& \delta_{1}=\lambda_{\max }(P)(1+\|D\|)^{2}+h_{M} \cdot \lambda_{\max }(Q)+h_{M}^{3} \cdot \lambda_{\max }(R) \\
& +\tau \cdot\left(\lambda_{\max }(S)+\lambda_{\max }(T)\right) .
\end{aligned}
$$


On the other hand, we have

$\lambda_{\min }(P) \cdot e^{2 \alpha t} \cdot\|\wp(t)\|^{2} \leq e^{2 \alpha t} \cdot \wp^{T}(t) P \wp(t)$

$\leq V\left(x_{t}\right) \leq \delta_{1} \cdot\left\|x_{0}\right\|_{s}^{2}$,

where $\wp(t)=x(t)-D x(t-\tau)$. From (9), we can obtain the following result

$\|x(t)\|=\|\wp(t)+D x(t-\tau)\| \leq\|D\| \cdot\|x(t-\tau)\|+\|\wp(t)\|$

$\leq\|D\| \cdot\|x(t-\tau)\|+\delta_{2} \cdot e^{-\alpha t}, \quad t \geq 0$,

where $\delta_{2}=\sqrt{\delta_{1} / \lambda_{\min }(P)} \cdot\left\|x_{0}\right\|_{s}$. Since $\|D\|<1$ and $\tau>0$, we can choose a sufficiently small positive constant $\xi=\alpha<-(\ln \|D\|) / \tau \quad$ satisfying $\quad\|D\| \cdot e^{\xi \tau}<1$. By the Razumikhin-like approach of [8], we obtain the following result

$$
\begin{aligned}
& \|x(t)\| \leq\left[\sup _{-h \leq \theta \leq 0}\|x(\theta)\|+\frac{\delta_{2}}{1-\|D\| e^{\xi h}}\right] \cdot e^{-\xi t} \\
& \leq\left[\left\|x_{0}\right\|_{s}+\frac{\delta_{2}}{1-\|D\| e^{\xi h}}\right] \cdot e^{-\xi t}, \quad t \geq 0 .
\end{aligned}
$$

This completes the proof.

Corollary 1: System (1) with $D=0$ is global exponentially stable with convergence rate $\alpha>0$, if there exist some $n \times n$ matrices $P, Q, R>0$, a matrix $U$, such that the following LMI conditions hold for all $i=1,2, \cdots, N$

$\Xi_{i}=\left[\begin{array}{ccc}\Xi_{11 i} & \Xi_{12 i} & \Xi_{13 i} \\ * & \Xi_{22 i} & \Xi_{23 i} \\ * & * & \Xi_{33 i}\end{array}\right]<0$,

where * represents the symmetric form in the matrix and

$\Xi_{11 i}=2 \alpha \cdot P+P A_{0 i}+A_{0 i}^{T} P+Q-e^{-2 \alpha h_{M}} \cdot R$,

$\Xi_{12 i}=P A_{1 i}+e^{-2 \alpha h_{M}} \cdot R, \Xi_{13 i}=A_{0 i}^{T} U^{T}$,

$\Xi_{22 i}=-e^{-2 \alpha h_{M}} \cdot\left[\left(1-h_{D}\right) \cdot Q+R\right], \Xi_{23 i}=A_{1 i}^{T} U^{T}$,

$\Xi_{33 i}=h_{M}^{2} \cdot R-U-U^{T}$

Table 1. Comparing Some Previous Results with this Paper
Remark 1: In [7], the slow variation condition $\dot{h}(t) \leq h_{D}<1$ is constrained in their considered systems. The constraint $h_{D}<1$ is not imposed on the results in this paper. By setting the matrix $Q=0$, Theorem 1 is still valid with unknown $h_{D}$.

Remark 2: By setting $\alpha=0$ and $R=0$ in Theorem 1, we can obtain the delay-independent stability result about system (1).

\section{NUMERICAL EXAMPLE}

Consider the system (1) with the following parameters: (Example 1 of [7])

$A_{01}=A_{02}=\left[\begin{array}{cc}-1 & 0 \\ 0 & -1\end{array}\right], A_{11}=\left[\begin{array}{cc}0 & 0.5 \\ -1 & 0\end{array}\right]$,

$A_{12}=\left[\begin{array}{cc}0 & 1 \\ -0.5 & 0\end{array}\right], D=0, N=2$.

By Corollary 1, a feasible solution of LMI (10) for $\alpha=0.3, h_{D}=0.1, h_{M}=0.4$ is given by

$P=\left[\begin{array}{cc}2.3029 & 0 \\ 0 & 2.3029\end{array}\right], Q=\left[\begin{array}{cc}1.5295 & 0 \\ 0 & 1.5295\end{array}\right]$

$R=\left[\begin{array}{cc}2.9047 & 0 \\ 0 & 2.9047\end{array}\right], U=\left[\begin{array}{cc}1.0457 & 0.0268 \\ -0.0268 & 1.0457\end{array}\right]$.

Some comparisons with the obtained results are made in Table 1. The results of this paper provide a larger allowable upper bound for time delay to guarantee the global exponential stability of system (1) with (11).

\section{CONCLUSION}

In this paper, the global exponential stability for switched neutral systems with mixed time delays has been considered. LMI and Razumikhin- like approaches are used to improve our results. The obtained results are less conservative via the numerical simulation.

\section{ACKNOWLEDGEMENT}

The research reported here was supported by the National Science Council of Taiwan, Republic of China under grant no. NSC 96-2221-E-022-012.

\begin{tabular}{|c|c|c|}
\hline \multicolumn{3}{|c|}{ Some Upper Bounds of Delay that Guarantee the Global Exponential Stability of the System (1) with (11) } \\
\hline Results & Convergence Rate $\boldsymbol{U}$ & Upper Bounds of Delay $\boldsymbol{h}_{\boldsymbol{D}}$ and $\boldsymbol{h}_{M}$ \\
\hline \hline$[7]$ & $\alpha=0.3$ & $h_{D}=0.1, h_{M}=0.4$ \\
\hline Our results & $\alpha=0.3$ & $h_{M}=0.44\left(h_{D}=1\right.$ or unknown) \\
\hline$[7]$ & $\alpha=0$ & $h_{D}=0.1, h_{M}=1$ \\
\hline \multirow{2}{*}{ Our results } & $\alpha=0$ & $h_{D}=0.1, h_{M}=3.58$ \\
\cline { 2 - 3 } & $\alpha=0$ & $h_{M}=0.68\left(h_{D}=1\right.$ or unknown $)$ \\
\hline
\end{tabular}




\section{REFERENCES}

[1] K. Gu, V. L. Kharitonov, and J. Chen, Stability of time-delay systems. Boston, Massachusetts: Birkhauser, 2003.

[2] J. K. Hale, and S. M. Verduyn Lunel, Introduction to functional differential equations. New York: Springer Verlag, 1993.

[3] V. B. Kolmanovskii, and Myshkis, A., Applied theory of functional differential equations. Dordrecht, Netherlands: Kluwer Academic Publishers, 1992.

[4] C. H. Lien, and K. W. Yu, "Stability criteria for neutral systems with time-varying delays and nonlinear uncertainties". J. Chin. Inst. Eng., vol. 30, pp. 517-522, May 2006.

[5] K. W. Yu, and C. H. Lien, "Stability criteria for uncertain neutral systems with interval time-varying delays", Chaos, Solitons Fract., (In Press)
[6] J. Liu, X. Liu, and W. C. Xie, "Delay- dependent robust control for uncertain switched systems with time-delay". Nonlinear Anal. Hybrid Syst., vol. 1, pp. 81-95, March 2008.

[7] X. M. Sun, J. Zhao, D. J. Hill, "Stability and $L_{2}$-gain analysis for switched delay systems: A delay-dependent method". Automatica, vol. 42, pp. 1769-1774, Oct 2006.

[8] C. H. Lien, K. W. Yu, Y. F. Lin, Y. J. Chung, and L. Y. Chung, "Exponential convergence rate estimation for uncertain delayed neural networks of neutral type", Chaos, Solitons Fract., (In Press)

[9] S. P. Boyd, L. El, Ghaoui, E. Feron, and V. Balakrishnan, Linear matrix inequalities in system and control theory. Philadelphia: SIAM, 1994. 